\title{
A Novel and Secure IoT based Cloud centric architecture to perform predictive analysis of users activities in sustainable health centres
}

\author{
P.K. Gupta * • B.T.J Maharaj · Reza \\ Malekian
}

\begin{abstract}
Diabetes, blood pressure, heart, and kidney, some of the diseases common across the world, are termed 'silent killers'. More than $50 \%$ of the world's population are affected by these diseases. If suitable steps are not taken during the early stages then severe complications occur from these diseases. In the work proposed, we have discussed the manner in which the Internetof-Things based Cloud centric architecture is used for predictive analysis of physical activities of the users in sustainable health centers. The architecture proposed is based on the embedded sensors of the equipment rather than using wearable sensors or Smartphone sensors to store the value of the basic healthrelated parameters. Cloud centric architecture is composed of a Cloud data center, Public cloud, Private cloud, and uses the XML Web services for secure and fast communication of information. The architecture proposed here is evaluated for its adoption, prediction analysis of physical activities, efficiency, and security. From the results obtained it can be seen that the overall response between the local database server and Cloud data center remains
\end{abstract}

\footnotetext{
* Corresponding Author
}

Department of Electrical, Electronic, and Computer Engineering University of Pretoria, Pretoria, South Africa, 0002

Tel.: +27 12 420-4033

Fax: +27 $12362-5000$

E-mail: pkgupta@ieee.org

Department of Electrical, Electronic, and Computer Engineering University of Pretoria, Pretoria, South Africa, 0002

Tel.: +27 12 420-4636

Fax: +27 12 362-5000

E-mail: sunil.maharaj@up.ac.za

Department of Electrical, Electronic, and Computer Engineering University of Pretoria, Pretoria, South Africa, 0002

Tel.: +27 12 420-4305

Fax.: +27 12 362-5000

E-mail: reza.malekian@ieee.org 
almost constant with the rise in the number of users. For prediction analysis, If the results collected in real time for the analysis of physical activities exceed any of the parameter limits of the defined threshold value then an alert is sent to the health care personnel. Security analysis also shows the effective encryption and decryption of information. The architecture presented is effective and reduces the proliferation of information. It is also suggested, that a person suffering from any of the diseases mentioned above can defer the onset of complications by doing regular physical activities.

Keywords Cloud centric architecture - Cloud health care architectures . Cloud security · eHealth · Health care applications · Health care framework . IoT Architecture $\cdot$ Sustainable health center

\section{Introduction}

Recent advancements in the field of wireless and multimedia technology as well as computing, hold out promise to perform real-time communication in the various user-based sectors of health, transportation, media, and education, etc. The vision of the Internet-of-Things (IoT) is one such and provides real time information to the connected users. The scope of the Internet-of-Things is broad and includes the living and non-living entities connected to each other in the network. Major IoT components include sensor devices, wireless communication devices, and application and services. A few additional components related to security and privacy can also be used. At a lower level these devices are networked with each other for timely processing of the information in real time and also categorized into various groups to manage the security related issues. In fact, the Internet-of-Things can be utilized with any of the sectors listed above, including eHealth. The meaning of term eHealth varies in terms of the source it is used for. World Health Organization (WHO) defines eHealth as the transfer of health-related information using electronic means[1]. Technologies like E-commerce and E-business play an important role in disseminating such information over the internet. Advancements in the field of wireless sensor network and cloud computing have also realized the vision of the sustainable health centers which monitor the health status and predict alerts in real time for both the health care personnels and users. Several organizations, like CISCO, General Electric etc. have expected that the IoT based health care market in future will become a $\$ 117$ billion market by year 2020 [2]. In the current work, the concept of Internet-of-Things has been used with the proposed cloud centric architecture to predict the users physical activities at sustainable health center of smart city. Most health care personnel purport that if an individual exercises regularly, then the complications and increase in the dosages of medications for diseases like diabetes, blood pressure, and heart issues, can be postponed accordingly. Fig. 1 represents the same information in which E shows the user group based on medications only, whereas E' shows the user group on medications as well as performing regular physical 


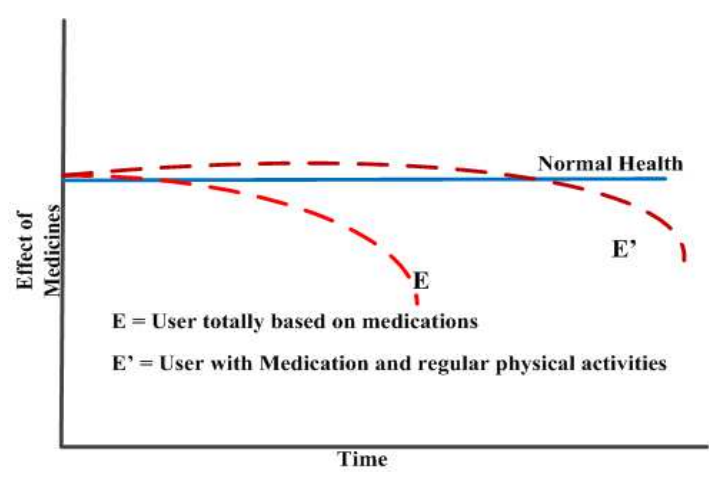

Fig. 1 Effect of medicines over time

activities. The E' user group lives a healthy life and can postpone the complications of the diseases mentioned above, whereas the E user group needs to increase their medication intake over a period of time to live a normal life. The proposed architecture monitors the users during their regular activities in these health centers and predict alerts to and from their health care personnel. Zhang et al., [3] explained the use of the wireless sensor networks in healthcare in the future from a ubiquitous perspective. They proposed a three-tier system of architecture for healthcare applications. They also stated that with the technological advancements, the field of medical informatics has focused more on as well as emphasized the use of the Smartphone's over wearable devices. They have realized several challenges in terms of information security, mobility control and applications while designing the applications. Chen et al., [4] have presented the vision of IoT from the perspective of China and specified that in "Remote medical monitoring" data can be collected from various sensor-like devices placed on an individual's body and once the data is processed advice can accordingly be given. In[5] Islam et al., have presented various aspects of the IoT-based health care technologies and stated that IoT can help any age group and address any disease in an innovative manner. They also focused on the various healthcare security challenges related to IoT and proposed an intelligent security model to mitigate these risks. They are confident that the use of the IoT and eHealth policies can lead to sustainable development in any country. In [6] Yang et al., have designed the home mobile healthcare system for wheelchair users. The proposed architecture utilizes the Smartphone for sending and receiving instructions from the source and sinks nodes. In [7] Amendola et al., have used the RFID based on wearable tags to identify the movement of the body parts like arms, legs etc.; in fact, they have used the IoT for monitoring the information collected on human lifestyles.

From the perspective of the health care personnel there are several advantages of real-time health monitoring of the users. This paper presents the use of technology like cloud computing and the Internet-of-Things and discusses the detailed implementation of the proposed architecture over the cloud cen- 
tric environment. This framework reduces the complexity and challenges as discussed by Zhang et al., [3]. Using the Clouds enables the efficient handling of processing the information and security. The users can move freely without requiring any wearable sensor on their body and of the necessity to use the Smartphone too is avoided..

This work makes the following contributions:

- A novel architecture has been proposed to monitor the users activities in sustainable health centers of a smart city.

- The proposed framework monitors the details of the basic parameters such as heart rate, distance, speed, and calories burned daily by a user, and stores this information only at the end of the session using either the public cloud or private cloud, respectively. Thus, the proliferation of information can be reduced.

- Health care personnel can access this stored information whenever required. In case of any irregularity is predicted in the users' activity or basic parameters then an alert is automatically sent to the health care personnel to initiate action.

- Provides better response time and security to the information stored.

Finally, this paper is organized into various sections where Section 2 summarizes about various works related to Internet-of-Things based architectures, and cloud based health care architectures proposed for eHealth. This section also presents summary about proposed health care applications that monitors the users activity continuously either using sensors or Smartphone's. In Section3, IoT based development architecture of proposed system has been presented. This section consists of overall development methodology presenting four layer architecture, user activity framework, and efficiency of proposed framework. In Section 4, we have presented the proposed cloud centric architecture representing the detailed connectivity, aligned with cluster of IoT based equipments to various types of clouds, and cloud datacenters. This section also presents major implemented cloud algorithms like predictive analysis mechanism, and Cloud security mechanism. Section 5, presents results and discussions and summarizes the results obtained for proposed architecture related to its adoption, predictive analysis of physical activities, efficiency, and security analysis. At last, we have concluded our work in Section 6.

\section{Related work}

The usage of IoT in the future lies in the hand of the technology and computing techniques, so that the information can be processed and provided in time to the user. A delay can result in the failure of the system. This section includes detailed focus on the work done related to the architecture of the IoT, health frameworks, health care frameworks and applications and Cloud based health care architectures. 


\subsection{IoT architecture}

Over a period of time different definitions and architectures of IoT have been proposed by various organizations. Chen et al., [4] have listed some of these proposed definitions which include the terms like "global infrastructure", "global network infrastructure" or "worldwide network" to refer to Internetof-Things. Here, one commonality in these definitions is that they build a bridge between the physical and virtual objects, which include communication technologies and data processing. These common features of IoT support its architecture. Chen et al., are also concerned with the high implementation cost of IoT because of the integration of data and use of private protocols by different organizations. They have focused on the need for an open and generic architecture of IoT. Yang and Zhang [8], as well as Ortiz et al., [9] have proposed an open and generic architecture of IoT, which is flexible, scalable, concurs with standard protocols, and can integrate multiple IoT applications. The China communication standard association has also proposed a generic IoT architecture consisting of three layers called sensing, network, and application [4]. Wan et al., [10] have discussed the IoT sensing framework which comprises four layers called sensing and information gathering, information delivery, information handling, and application. All of these layers ensure privacy and security throughout the use of the information. In [11] Domingo has proposed the three-tier IoT architecture for disabled individuals and includes layers like perception, network, and application. The proposed architecture was implemented for different application scenarios like shopping, school, and smart homes. Similarly, several more IoT architectures [12]-[16] have been proposed and discussed for various scenarios.

\subsection{IoT based health care frameworks}

With the rising demand and great future possibility of the implementation of the IoT in eHealth, several frameworks have been proposed for real time monitoring of the users and to provide support if required. In [7] Amendola et al., have implemented the RFID technology with the sensing or physical layer of the IoT architecture to monitor user health and activate remote assistance when required. They have recorded the users personal health using the various body-centric RFID tags such as the wearable and implantable tags. Authors have also discussed the scenarios where these tags are sufficient to collect the data but lack the implantation details and the results make this work appear weak. They have concluded that there is great need to boost the data transfer rate of the readers, different softwares and devices to take timely action. In [17] Santos et al., has also proposed an RFID based M-health care system using the IoT based connected devices. They have focused on the enhancement of the health services using IoT and RFID technology. In [6] Yang et al., have designed a prototype of intelligent home healthcare monitoring system for disabled individuals. This prototype is implemented using 
the wireless body sensor networks such as wireless heart rate sensor, wireless pressure sensor, and home environment sensing. These sensors have been presented in their mHealth framework. They used the Smartphone to deploy the prototype for sending and receiving the commands. Here, the use of the Bluetooth v 2.0 limits and slows down the functioning of the prototype; the security aspects related to data have also not been discussed. In [3] the work presented by Zhang et al., discusses the use of the wireless sensor network in the health care systems. They have designed a three-tier framework to implement the proposed system. Authors have discussed the different aspects of data communication standards like IEEE 802.11-2012 standard for WLAN, IEEE 802.11-2011 standard for cognitive radio, IEEE 802.20 standard for high velocity mobile broadband system, IEEE 802.16-2009 standard for WiMAX and mostly used by telemedicine, and IEEE 802.15.1-2005 standard for Bluetooth. They have identified that the IEEE 802.16 standard can be used throughout the healthcare systems because it is collision free and provides high QoS in real time for various medical services. The authors have not discussed the security aspects of the proposed framework. In [18] Mitra et al., have proposed a KNOWME body area sensing platform for end-to-end communications and used the wearable sensors and Smartphone for collecting and transferring the captured information. The proposed KNOWME platform comprised is composed of four layers for performing the tasks and reduces the complexity of the communications by using the code-division multiple access system (CDMA). The authors have mainly focused on the sensor management, and maintaining the buffers as well as emphasized the need for revisiting the wireless communication standard for eHealth.

\subsection{Health care applications}

Rapid growth of health care related applications simplifies tasks deployment, collection and performing data analysis easier. Advances in sensor devices also lead to this development of new applications to be used by eHealth. Mobile healthcare which is also known as mHealth has only become possible because of this advancement in sensors technology and represents the new opportunities for mHealth applications. According to ITU, there are 5.9 billion mobile subscribers around the world and most of the organizations and researchers want to utilize this market as emerging mHealth market for health care delivery [19]. Wide range of mHealth applications can be found for monitoring of Diabetes [20], Blood Pressure [21], Heart rate [22], Physical activity [23], and Anti-Obesity [24] like areas. There are varieties of health care applications available for various types of Smartphone, Tablets, and iPad like devices and are known as BlueBox [22], WIHMD [21], AppPoint [25], HeartToGo[26], Instant heart rate [27]. Though various types of applications can be find over internet but somewhere these applications lack in security and privacy, reliability, efficiency, and acceptability. Moreover, these applications are designed for Smartphone like devices where battery life is another issue. Here, Fig- 
ure 2 represents the various types of health care applications designed for Smartphone's. These applications continuously checks for the incoming data obtained by sensor for activities like heart rate, ECG, pulse, blood pressure, and blood sugar levels of user and keep informing to the health care personnel in case of any emergency.

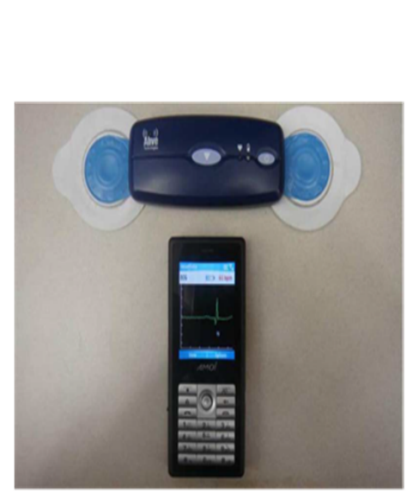

(a)

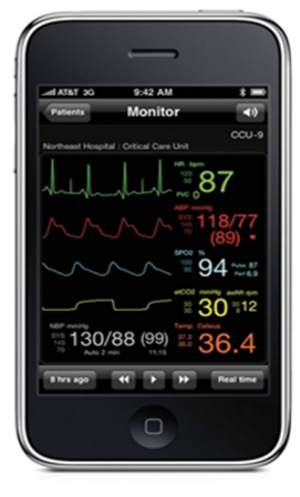

(b)

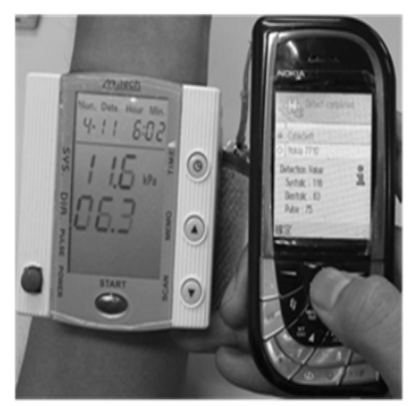

(c)

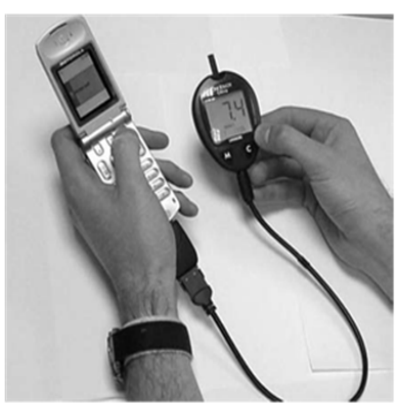

(d)

Fig. 2 Health care applications uses Smartphones to monitor (a)ECG, (b)Blood pressure, heart rate, SPO2, and body temperature (c) Blood pressure and Pulse (d) Blood sugar level.

\subsection{Cloud based health care architectures}

As we have seen, by introducing IoT in health care leads to development of new frameworks, and applications by researchers and organizations. Further, integration of clouds with IoT based frameworks could provide several benefits 
of enhanced information security, quick access of records, and energy savings over traditional existing health care frameworks. In [28] Kumar et al., have proposed a RFID based intelligent authentication scheme in the health care using vehicular cloud computing. The authors have placed various tags and readers from users to road side units. Communication between these tags and readers are secured by elliptical curve cryptography based key generation algorithm. Authors have evaluated the effectiveness of proposed scheme using various parameters. In [29] Hassanalieragh et al., have presented the integration guidelines for remote health monitoring in to medicinal practice. They have used smartphones as concentrator in IoT infrastructure and for aggregation of data, cloudlet's or clouds have been used. It is also realized that data processing could be done more efficiently using clouds rather that cloudlet's and wearable sensors have been used to collect the information. Authors have performed two-three days of continuous physiological monitoring using these sensors and collected related physiological parameters to update the relevant health database. In [30] Seales et al., have proposed architecture for content centric networking for Health-IoT. This networking has several benefits and integrates health services and sensors, and clouds in Health-IoT. Proposed PHINet architecture automatically record the body data from user body sensors and further update the database accordingly. Use of clouds in this architecture makes data easy to handle for analysis of both user and health care personnel. Wan et al., [10] have implemented the platform production services using IoT and inter cloud computing architectures. Proposed framework was used by vehicular networking applications. Authors have evaluated the performance of proposed system using probabilistic theory.

\section{Proposed IoT based Development Architecture}

From the literature reviewed, we can find that there is still a lot of possibility for the improvement and optimization of the proposed and implemented frameworks until now. Most of the proposed health care frameworks are over optimized as they generate large amounts of data, and continuously send alerts to the users and health care personnel, which are of no use. Most of these frameworks and applications are designed to use the Smartphone for monitoring and capturing of data. In such a scenario, the continuous connectivity of the Smartphone devices is questionable. Keeping these shortcomings a priority, we have proposed the IoT based development framework as shown in Fig. 3 which consists of the evaluation, implementation, feedback and security layers in the IoT environment for a cloud centric communication between the user and health care personnel. The development framework simplifies the data acquisition and data analysis tasks and also reduces the complexity of implementation and connectivity of the devices at a physical level.Similar to several other frameworks, this proposed architecture is not Smartphone centric and keeps tracking the user's activity in the sustainable health centers on a regular basis. 


\subsection{Development methodology}

The methodology behind the application development is to implement the IoT architecture layers for cloud centric communications. This methodology proposes four different layers which are discussed later in this section and claims to provide simplified and faster communication between the user and health care personnel. This methodology also reduces the amount of data necessary to be stored for analysis purposes and requires regularity in the activity of the users so that the health care personnel can be kept informed on the users' health and activity status.

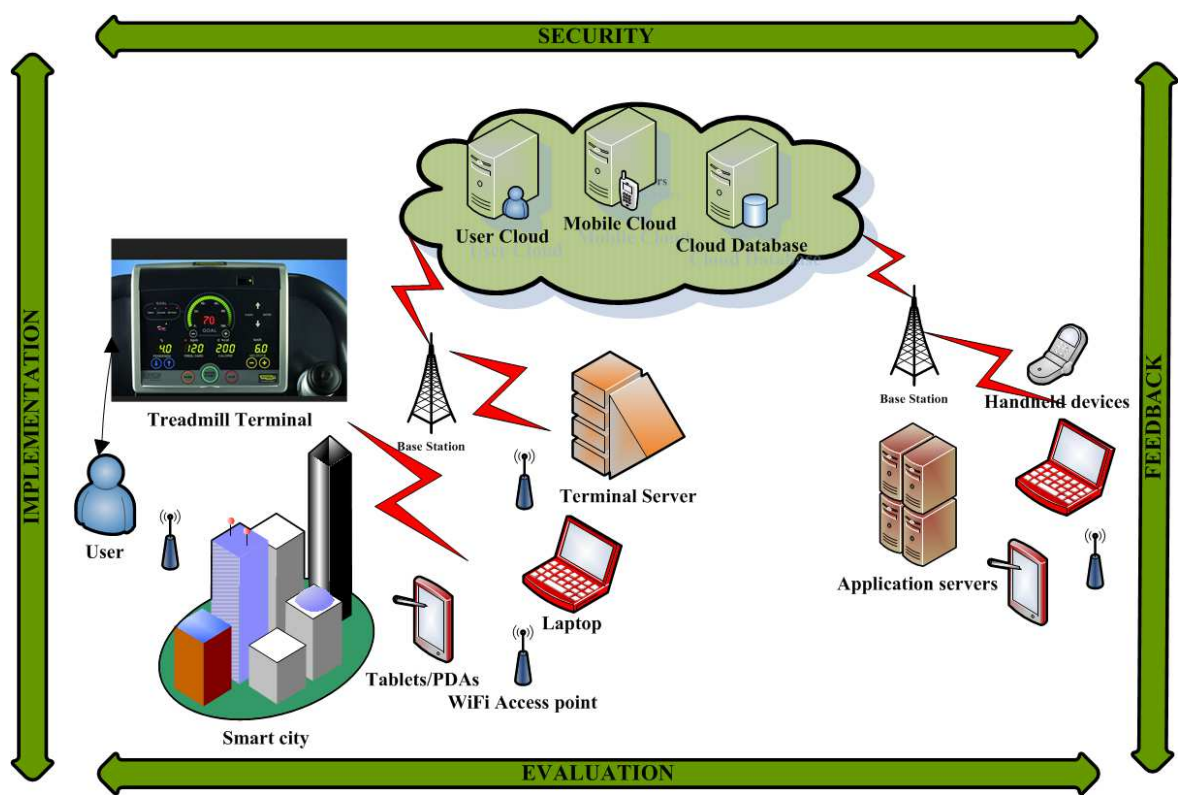

Fig. 3 Proposed IoT based development architecture for Cloud centric communication

- Implementation layer simplifies the overall architecture from the capture of information to its storage and analysis. This architecture uses the embedded sensors of the equipment rather than any wearable sensors by the user. This layer implements an application for monitoring the health of each individual user. The application in turn transfers the collected data to the required cloud from where it can be accessed by the health care personnel.

- Evaluation layer represents thorough usage of the implemented application by the user. Issues reported by the user and health care personnel related to the use of application or communications are addressed. This layer performs its functions throughout the utilization of the application. 
Here, the main focus is to ensure a reduction in the complexity and its adoption to improve the health care services.

- Feedback layer uses the outputs of the evaluation layer which are used to modify the application accordingly. After each evolution cycle of the implemented application, reported modalities are rectified, which helps in improving the performance of the overall proposed architecture.

- Security layer focuses on the security related issues during storage and communication of the information. This layer also provides its functioning for cloud centric environment for various types of cloud servers like User cloud, Mobile cloud and Cloud database. The purpose of this layer is to ensure the security of user information for which he/she has subscribed.

The role of each layer is to ascertain whether they meet the desired goals stated for them or not. These desired goals may be different for each layer and focus on the need of the user and health care personnel. These goals also ensure the overall quality of the system, and the collected information could be used to generate the visual results and alerts regarding the users' health. This information is stored confidentially with various types of clouds by implementing appropriate security mechanism.

\subsection{Application architecture}

Application architecture for the IoT based system proposed above reduces the complexity of the information storage, communication and enhances the performance of the overall health care system. Fig.4 introduces the multilayer application architecture which includes the IoT sensor devices, Treadmill equipment, application servers, base stations, and handheld devices like PDAs, Tablets, notebooks etc. at the bottom. These interconnected devices communicate with the user interfaces designed with JAVA. These user interfaces are responsible for capturing the information from the connected sensors of the treadmill equipment at the end of the user session and any further update of this information with the cloud servers. On the other hand, health care personnel can utilize these user interfaces to know the health status of the users and advise them accordingly. These user interfaces are further connected via the middleware with many Cloud Servers and support the inter cloud communication with Cloud Database. In middleware technologies, XML Web-Services can be used to carry secure information from these interfaces to the cloud and can also communicate with the required database for reporting to the user's request. User database is defined and designed using the star schema for reporting purposes. This multi-dimensional star schema related to many relations including authentication and authorization, and processes the required information accordingly. Standard SQL queries have been used to fetch and insert records from the multi-select relations. It is also ensured that the information flow is consistent throughout the layers and appropriate Cloud security mechanisms like AES and RSA have been implemented for encryption and decryption of the information. 


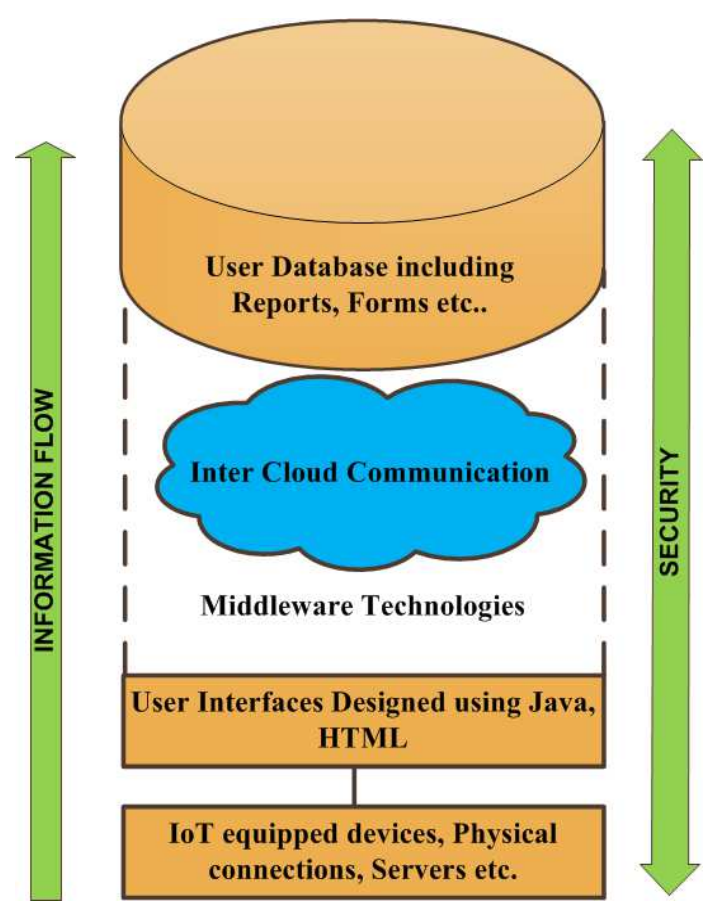

Fig. 4 Application Architecture

\subsection{User activity framework}

This is an interactive user activity framework which keeps both the users and health care personnel updated. Here, user of the system has been considered as a smart user and performs health related activities in sustainable health centers. These sustainable health centers have equipment fitted with sensors which are sufficient to capture the basic parameters related to the users' health activities such as average heart rate, total calories burned, total distance and average speed. This framework captures the information required once the user completes the exercise. Regular activities in these health centers are believed to be able to keep a user fit rather than on the heavy dependence on medicines. Health care personnel also suggest the same,and encourage regular physical activity to keep healthy for a longer time and avoid overdose of the prescribed medicines. Normally, brisk walking is recommended for users with diseases like diabetes, blood pressure, and heart patients and the treadmill equipment can be utilized for the same purpose. The proposed activity framework monitors the user's activity on a regular basis and updates the captured information to the required cloud. In case the user does not perform an activity for a specific time period then this information will also be updated in the cloud database and an alert will be sent to the respective health care personnel, who can 
further communicate with the user regarding understanding the status of his health.

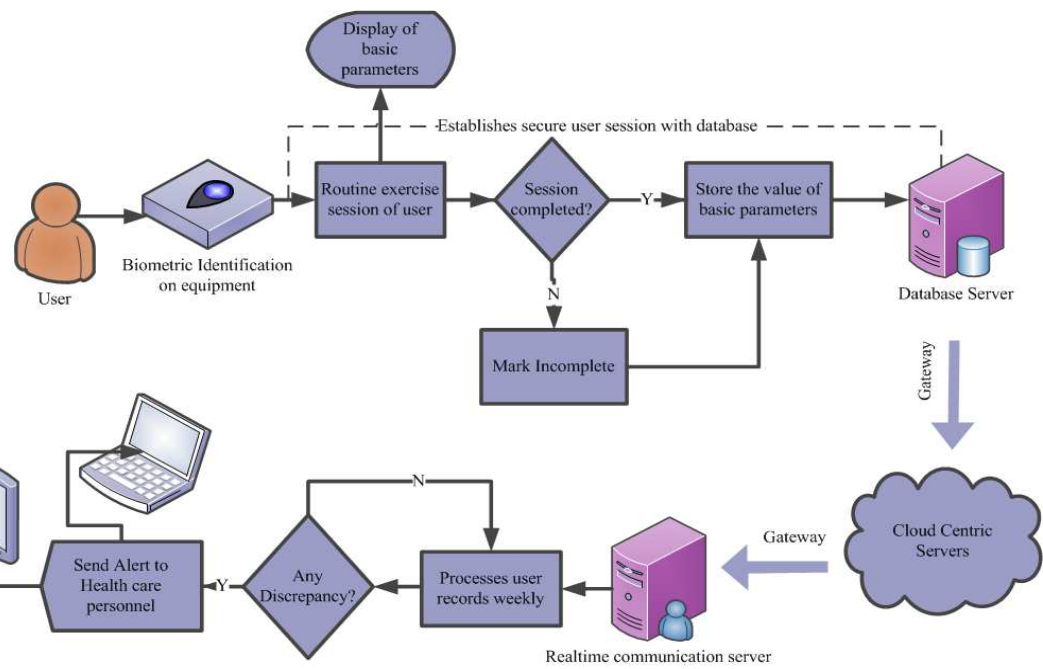

Fig. 5 User activity framework

Fig. 5 represents the user activity framework from the beginning and until end of the physical activity in a sustainable health center. As the biometric identification and authentication are completed for the user, a secure session gets established with the local database server to monitor the user's activity on that equipment. Here, it is considered that the equipment have embedded sensors which can monitor and transfer the details of the basic parameters like heart rate, total calories burned, total distance and average speed to the connected local database servers. As soon as the physical activity session is over, the equipment stops and the respective values of the basic parameters are stored in the local database server of the sustainable health center. In case the users terminate the session in the middle of the physical activity then that session will be marked INCOMPLETE and the respective values of the basic parameters are stored in the database. In the next step of this framework, the information stored at the local level is transferred via the gateway to the various Cloud servers. These Cloud servers keep the information up-todate and secure. Later on, this stored information is picked up by the real time communication servers located geographically with the different health care personnel. These real time communication servers process the record thus obtained and relate them to the user on a weekly basis to produce the reports accordingly. If the user regularly terminates the physical activity sessions, before the prescribed activity time for a particular equipment or is absent from the sustainable health center then an ALERT message is sent to the user's health care personnel either in the form text message or an Email alert. 


\subsubsection{Efficiency analysis of the proposed framework}

In this section we have analyzed the efficiency of the proposed framework using processing response time i.e. when the health issue had been noticed and when the advice to the user was provided in real time environment. As stated earlier, the proposed framework captures the basic health parameters of a user and if any health related issue is observed then the CollectBasicParameters() method will provide the details related to that particular user. Health care

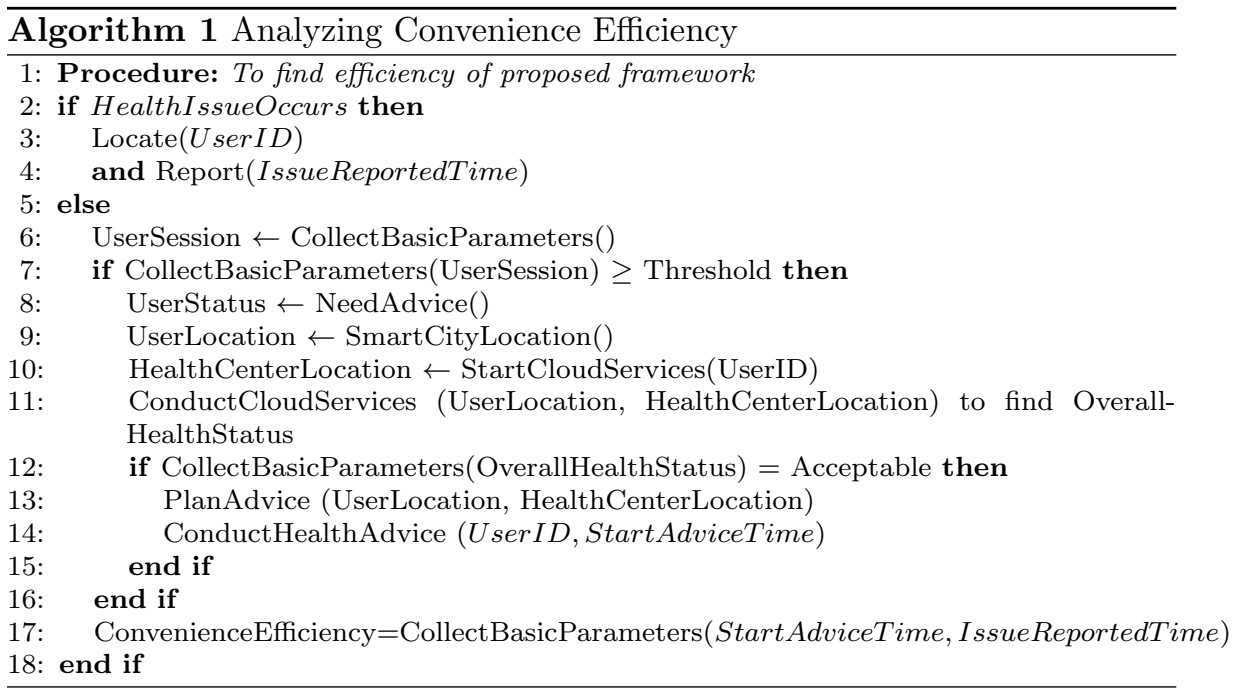

personnel can study these stored basic parameters, overall physical activity sessions and the health status of the User to accordingly provide advice in real time. Cloud based XML services are used to find the locations of the User and HealthCenter and to access the OverallHealthStatus. Function ConductCloudServices is used to obtain these details. In casethe overall health status of the user is found to be acceptable then function PlanAdvice is used to support the user who requires attention. Therefore, the function, CollectBasicParameters can provide the rough estimated efficiency of the proposed framework. If $T_{a}$ is the start of the advice time and $T_{i}$ is an issue reported time then cost function $C$ can be described with certain assumptions, as follows:

$$
C=\operatorname{Min}\left(T_{a}-T_{i}\right)
$$

Assumptions:

- User perform regular health checkup at least once in 6 months and keep the records updated with his cloud database.

- User with critical disease can be put on high alerts and may require frequent advice. 
4 Proposed Cloud centric architecture

The proposed cloud centric architecture reduces the overall complexity of the implementation of the system. It implements various security measures at each step of the communication of the information. As shown in Fig.6, the initial level collects and stores the information for each user from the sustainable health centers into their database server at the local level. The information collected represents the daily values of the basic parameters the specific user has worked out. To communicate this information thus gathered over the cloud the XML Web services have been used as they are considered secure and faster. From the initial level, the information is collected from the various database clusters distributed to the cloud centric data center. These data centers also ensure the security of the stored information and further disseminates the information to the public and private clouds. The information stored in these clouds depends on the type of service and level of security required by the subscribed user.

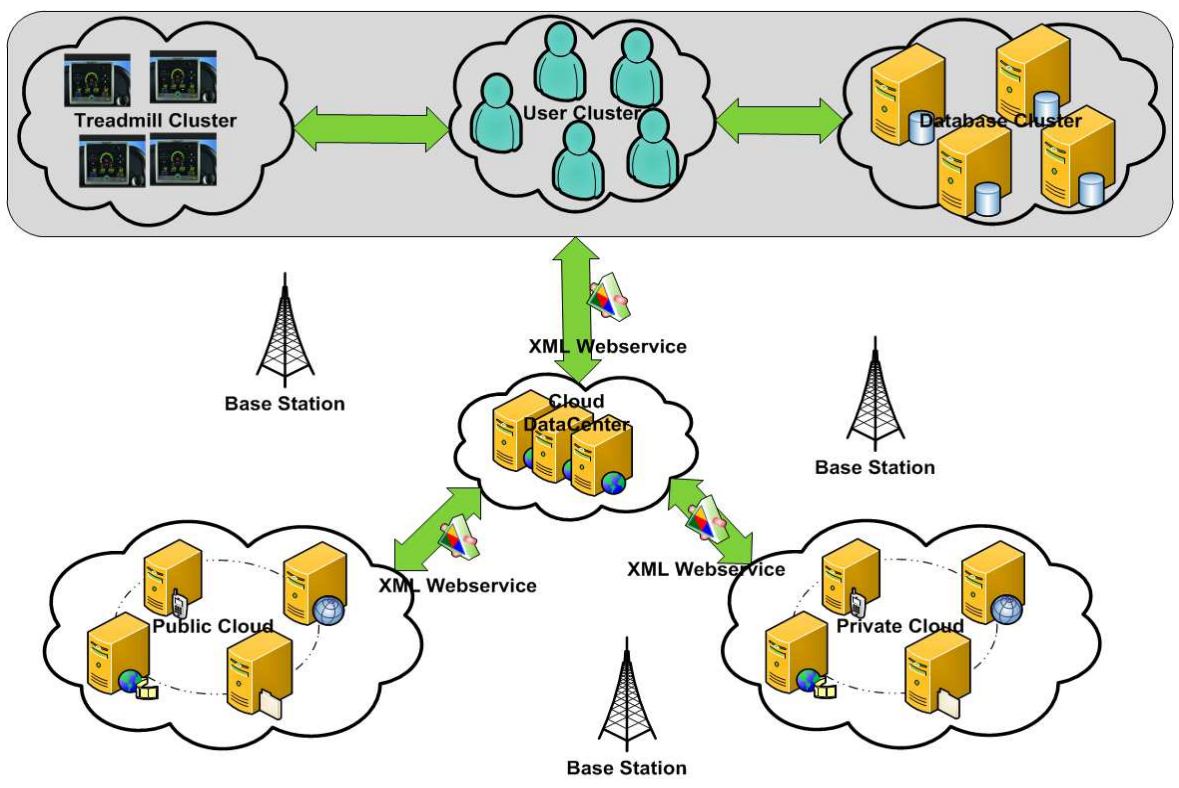

Fig. 6 Cloud Centric Architecture

Private Cloud - claims secure and prompt communication from the initial level to the health care personnel. Users can be subscribed to faster and secure communication by selecting this cloud. Users suffering with critical disease must subscribe to a private cloud for continuous monitoring by their health care personnel. If the system detects any emergency then an alert message 
is sent to all the attached devices of the health care personnel to handle the situation efficiently.

Public Cloud - compared with the private clouds, these clouds are freely available to access and store information. Here, the stored information is processed by the health care personnel on a weekly basis or whenever it is required. Usually, users with good health status subscribe to this cloud. Handling of alerts is similar to a private cloud.

Both private and public clouds are equipped with a Mobile information server, content management server, file server, and streaming media servers. These servers continuously exchange information whenever required with their respective clouds. While implementing this architecture, different algorithms are used with different types of clouds to maintain the integrity, consistency and security of the user's information.

\subsection{Cloud centric Algorithms}

In this study the major implemented algorithms which perform their functioning in a cloud centric environment have been presented. Algorithm 2 reveals the overall cloud centric mechanism using three levels viz., Initial, Cloud data center, and Alert mechanism. The initial level is associated with the user identification and authentication and supports the IoT based connectivity of the various equipment of the sustainable health center with local database servers. Here, the example of the Treadmill machine is used,where the user selects a maximum exercise time $(T)$ of 20 minutes. If the registered user in the local database is absent or terminates the session in between, then an Incomplete or Absent message gets accordingly stored in the database. The value of the basic parameters gets stored after the end of each session with the local database servers. Once the value of the basic parameters is stored, it is then sent to the Cloud data center using the appropriate XML Webservice. The Cloud datacenter is connected to clusters of local database servers located within the smart city. As soon as the these cloud data centers receive the information, the rules defined for the aggregation of information are applied and stored with the public and private clouds, accordingly. Finally, in the section of alert mechanism, the type of user $U$ is recognized and an alert is sent whenever any of the values of the basic parameters exceeds the defined threshold level $(\tau)$ for that user. Here, Algorithm 3 shows the implemented cloud security mechanism for a private cloud $(\Xi)$ and public cloud $(\Upsilon)$. Based on the user type $U$, the type of cloud and its security mechanism are selected. Private cloud service providers are free to implement any security algorithm like Rivest, Shamir, and Adleman (RSA), DNA, and Bcrypt, etc. Here we have implemented the asymmetric and public key based RSA algorithm. For public cloud, we have used the Advanced Encryption Standards (AES) algorithm for implementation which is a symmetric cipher and iterative algorithm. 


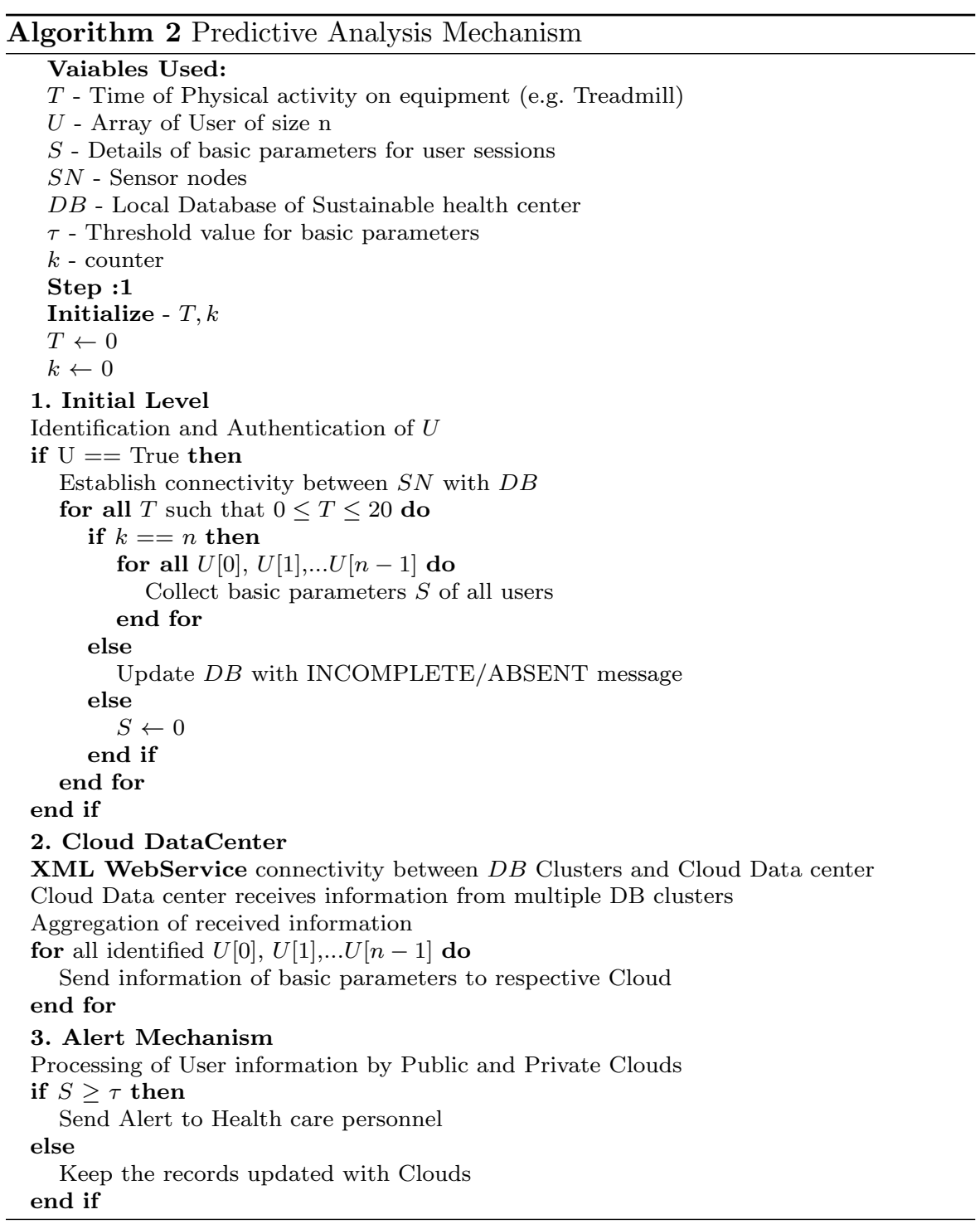

\section{Results and Discussion}

In this Section, the results obtained after implementing the proposed framework and the algorithms discussed for the various scenarios have been discussed. For simulations of the scenarios proposed MATLAB v7.10, 32-bit editionhas been used. A graphical user interface (GUI)has been written using JAVA, which incorporates the XML WebService for sending and receiving the information in a cloud centric environment from both the user and health care personnel. We have evaluated the proposed cloud centric system architecture 


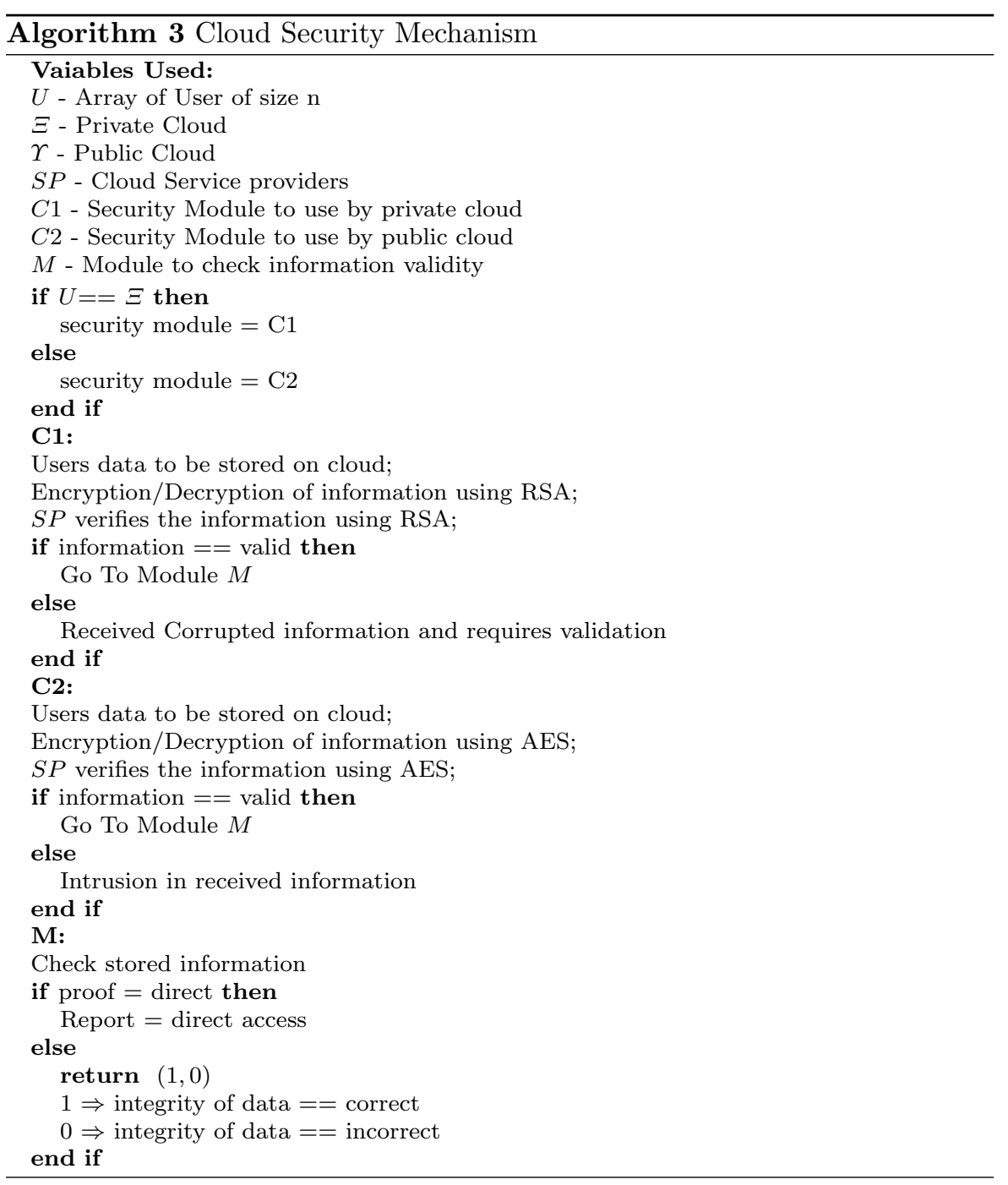

and user activity framework for their adoption, prediction analysis of physical activities, efficiency, and security purposes.

\subsection{Adoption}

In the case of the adoption of the proposed framework, it was found that the web-based health care services providing the applications are inadequate to track the user's health status on a regular basis. From a survey of the literature it was found that the current trend is more towards the use of Smartphone like devices to monitor the status of the user's health. Once the monitoring ap- 
plication stores the information using the Smartphone, it then provides it for use by the web-based applications, and then seeking the advice of the health care personnel becomes a big challenge in itself. This also downgrades the performance of the overall system as well. The proposed Internet-of-Things based platform and cloud centric architecture provides both with faster communication of information and security. The users are no longer required to focus on their Smartphones for storage of the values of the defined basic parameters. The proposed architecture is intended to change the way of eHealth, as it focuses on the regular activities of the users in sustainable health centers. In Fig.7,the overall response time for sending information from a cluster of local database servers to a Cloud data center has been presented.Simulated results show that the response time remains almost constant,which is less than 0.02 sec, with the increase in the number of users from 50 to 5000. These results are preliminary and highlight the reduction of complexity over the traditional web-based systems.

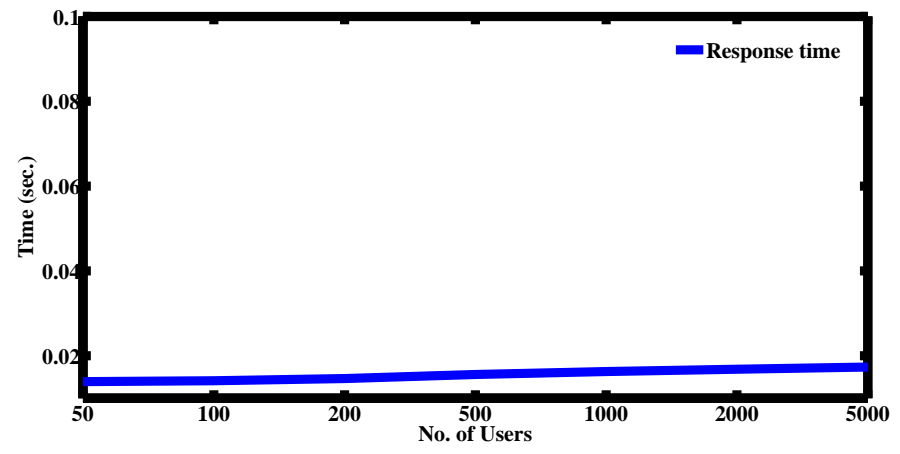

Fig. 7 No. of Users vs. response time

\subsection{Predictive Analysis of physical activities}

The proposed framework presents the ease of interface to both the health care personnel and users. While performing a physical activity in a sustainable health center it represents no interface to the user except for identification, authentication and establishing the session with the local database server which is responsible for storing the values of the basic parameters aa the user completes the session. Fig. 8 represents the values of the basic parameters stored after 20 minutes of each physical activity session by the users. Fig. 8 shows the various scatter plots for the distance, calories burned, heart rate, and speed with respect to the age of the users. Health care personnel can thus define the daily maximum threshold value of these parameters for their users individually and further predictive analysis can be performed accordingly. Here, 
Figure 8 (a) represents the threshold value for distance $>=4.5 \mathrm{~km}$, Figure 8(b) represents the threshold value for the calories burned $>=340$, Figure 8 (c) represents the threshold value for the heart rate $>=170 \mathrm{bpm}$, and Figure 8 (d) represents the threshold value for speed $>=13 \mathrm{~km} / \mathrm{hr}$. While performing prediction analysis, the defined threshold values of these basic parameters are checked and if any of value exceeds the limit then an alert is sent to respective health care personnel to initiate the necessary action. Also, if a user misses the physical activity session for a whole day or terminates the session in between, then too a message "INCOMPLETE/ABSENT" gets stored into the database.

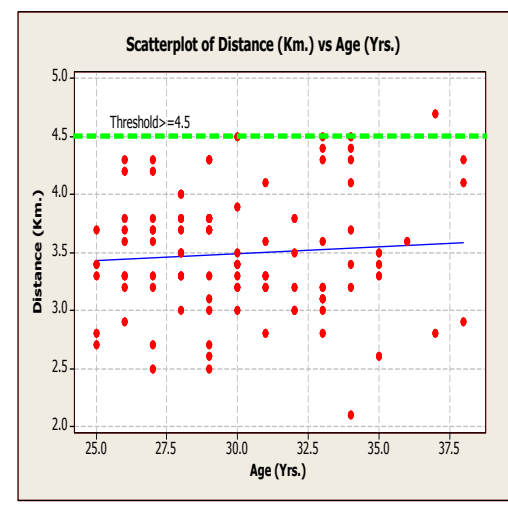

(a)

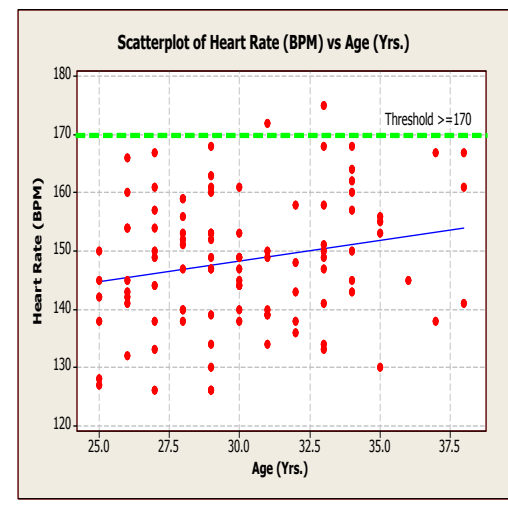

(c)

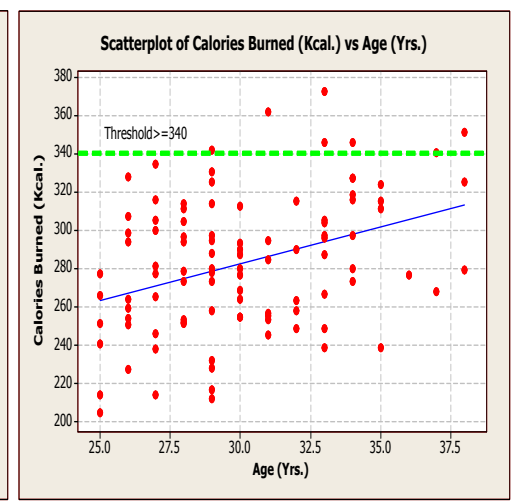

(b)

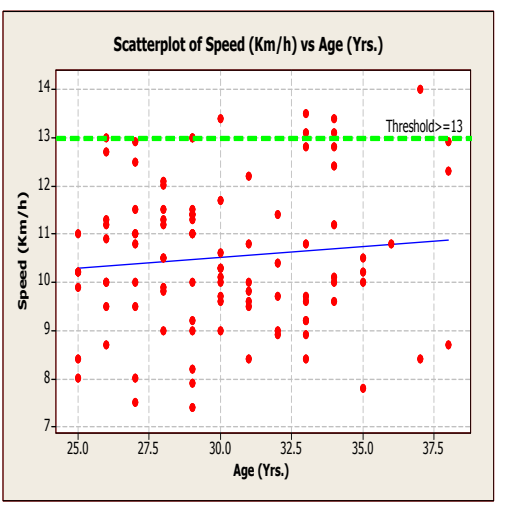

(d)

Fig. 8 Scatter analysis of users on treadmill (a) Distance (km.) vs. Age (Yrs.) (b) Calories burned (Kcal.) vs. Age (Yrs.) (c) Heart rate (bpm) vs. Age (Yrs.) (d) Speed (km) vs. Age (Yrs.). 


\subsection{Efficiency}

The efficiency of the proposed framework is evaluated using the total communication time, and end-to-end delay.

- Total communication time represents the total time taken for the storage of information from the local database server to the Cloud data center and further to the public or private clouds. This time can exceed if there is any alert sent from the public or private clouds to the health care personnel. Here, Equation 2 shows the computation of the total transmission time where $n$ represents the number of clusters, $t$ is the time, and $w$ the total offloading iterations, and $x$ total number of alerts.

$$
\left.\left.T_{\text {Time }}=\sum_{i=1}^{n}(t(w))+t(x)\right)\right)
$$

If there is no alert then the value of $t(x)=0$. Then Equation 2 will become:

$$
T_{\text {Time }}=\sum_{i=1}^{n}(t(w))
$$

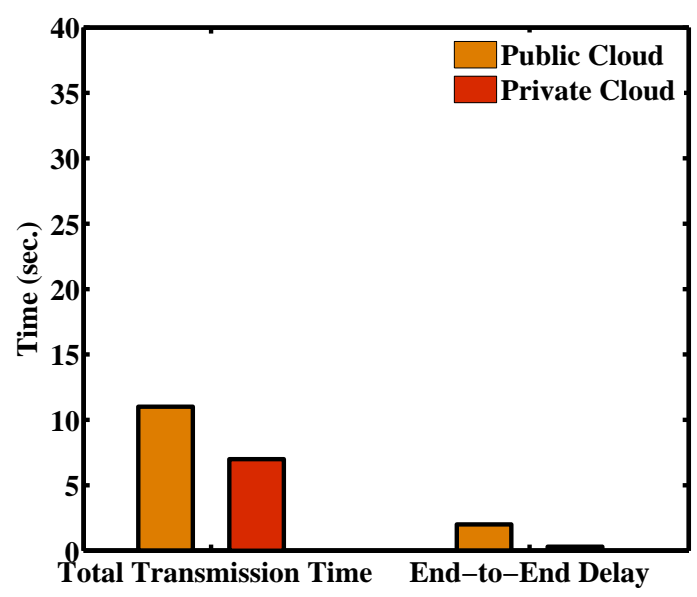

Fig. 9 Efficiency of Cloud centric framework

Fig. 9 represents the total transmission time for both the public cloud and private cloud. Here, the value of $T_{T}$ ime reaches up to 12 seconds for the public cloud and 7 seconds in case of the private cloud. The higher value of $T_{T}$ ime for the public cloud shows that more users are connected with it, as the services are not payable to this cloud. 
- End-to-End Delay represents the information delay time between the nodes. This can be computed by subtracting the minimum time of communication from the total time of communication. Its minimum value shows the early arrival of information at the destination node.

$$
\begin{gathered}
T_{\text {delay }}=T_{\text {Time }}-T_{\text {minimum }} \\
T_{\text {minimum }}=f\left(T_{\text {Time }}, \text { minimum }\right)
\end{gathered}
$$

In the case of the end-to-end delay, as shown in Fig. 9 the value of $T_{d}$ elay is at its minimum for the public cloud and almost negligible in the case of the private cloud.

\subsection{Security Analysis}

Cloud centric computing places various security threats on the information to be communicated or stored. These threats could be related to privacy, integrity, trust, or authentication of information. These threats become more vulnerable to danger when a user is associated with the public clouds. Private cloud provides different type of security mechanism which can be implemented throughout the communication for individual user on the basis of "Pay-peruse" model. In this section, two different algorithms RSA and AES have been implemented for private cloud and public cloud respectively. Implementation of the AES provides confidentiality to information in the cloud environment. This algorithm implements the variable size of the key length with a fixed data clock length of 128 bit. From Fig. 10 (a) it can be observed that the average encryption time taken for the data block varies with number of bits. It also increases if the mixed type of data is used that may include text and data. Fig. 10 (b) represents the average decryption time which is almost constant for the different numbers of bits of key lengths. Here, the execution time is measured in microseconds and depends on the length of selected text. The RSA algorithm provides several benefits over other existing algorithms, which isthe reason for the Private cloud service providers choosing to use it. TheRSA algorithm is an asymmetric and public key algorithm wherethe user's confidential information consists of two prime numbers $p$ and $q$. Here, the computation of the key depends upon the values of $p$ and $q$ and the value is represented by the product of $p$ and $q$. For the experiments, we selected the values of $p=2$ and $q=3$, , and from Fig.10(c) it can be observed that the average encryption time of the RSA increases with the increase in the text length. Fig.10(d) shows the time taken to decrypt the information which varies with the increase in the number of bits. Here, it is also observed that the RSA is more secure, which is the reason why it takes a little more time while encrypting or decrypting the information when compared with the AES, where the decryption time is almost constant. 


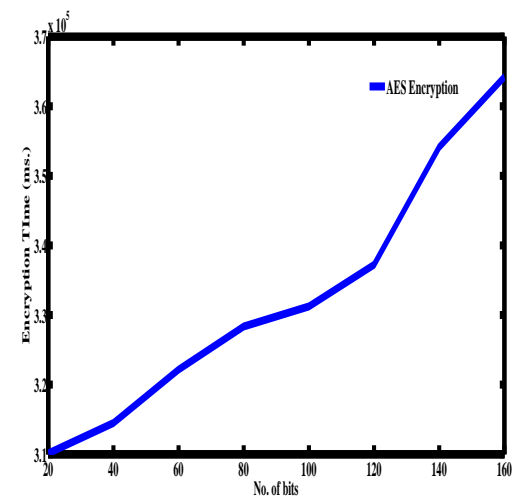

(a)

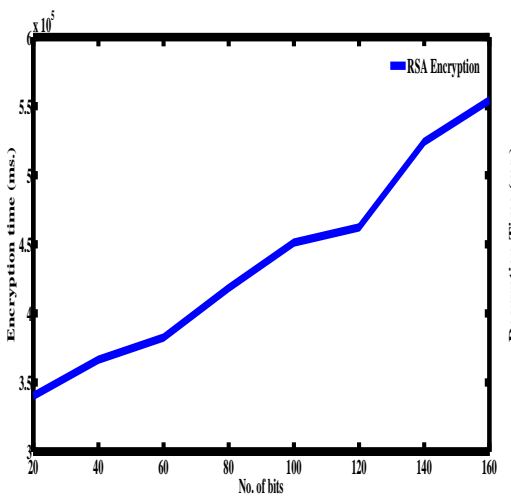

(c)

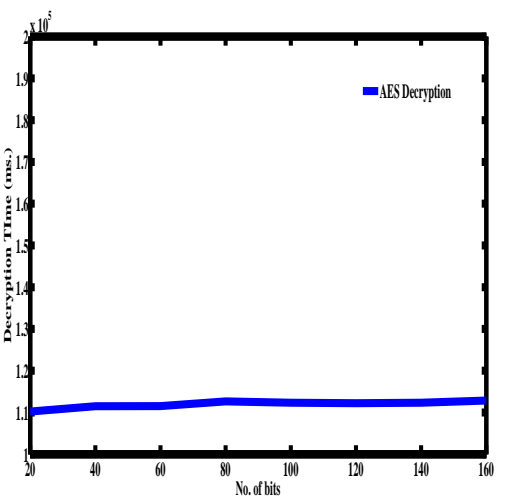

(b)

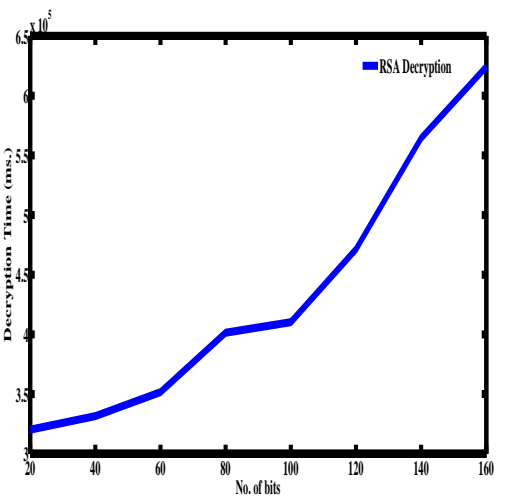

(d)

Fig. 10 Encryption and Decryption time vs. No. of bits (a) AES Encryption (b) AES Decryption (c) RSA Encryption (d) RSA Decryption.

\section{Conclusion and Future work}

At present, the health care sector is getting a boost with several advanced technologies like the Internet-of-Things and Cloud Computing which has enabled to perform prediction analysis in real time. Different varieties of advanced applications are being designed for Smartphone like devices to monitor the user activity and health status throughout the day. From our literature survey, this can be find that most of these applications have been found to suffer from the optimization issue and continuous proliferation of information which can be difficult to predict and store. The proposed IoT based cloud centric architecture simplifies several of these issues and stops the proliferation of information. 
This framework can be used by any age user group. For the proposed framework, we have evaluated on the basis of its adoption, predictive analysis of physical activities, efficiency, and security. There was also the understanding that to minimize the overall cost there should be a minimum time delay between the advice time and issue reported time. This can be observed from the results obtained for the end-to-end delay for the proposed framework. Predictive analysis of the physical activities represents the results collected in real time using the sensors for the various users on the treadmill for a 20-minutes time duration. These results also present the common threshold value for all the users,although the health care personnel can define it separately for the individual users. The results for the security analysis reveal the thorough encryption and decryption of information using the AES and RSA algorithms for the public and private clouds, respectively. Overall, the architecture presented and discussed is more robust and secure in nature, although a lot of work could still be done in the future for its improvement. We will consider the issue of load balancing and information distribution throughout the Cloud servers, implementation of more security algorithms like DNA encryption, XML DNA Encryption, Bcrypt etc., and extending the proposed framework to include more health parameters and activities.

\section{References}

1. Lake D., Milito R., Morrow M. and Vargheese R., Internet of Things: Architectural framework for eHealth security, Journal of ICT Standardization, vol.1, 2014.

2. $\$ 117$ Billion Market For Internet of Things In Healthcare By 2020 http://www.forbes.com/sites/tjmccue/2015/04/22/117-billion-market-for-internetof-things-in-healthcare-by-2020/\#4540fb042471 (Accessed on February 17, 2016)

3. Zhang Y., Sun L., Song H. and Cao X., Ubiquitous wsn for healthcare: Recent advances and future prospects, Internet of Thing, vol.1, no.4, pp.311-318, 2014.

4. Chen S., Xu H., Liu D., Hu B. and Wang H., A vision of IoT: Applications, challenges, and opportunities with china perspective, Internet of Things, vol.1, no.4, pp.349-359, 2014.

5. Riazul Islam, S.M. Kwak, D. HumaunKabir, M., Hossain M. and Kwak K.S., The internet of things for health care: a comprehensive survey, IEEE Access, vol. 3, pp.678-708, 2015.

6. Yang Lin, YanhongGe, Wenfeng Li, WenbiRao, and WeimingShen, A home mobile healthcare system for wheelchair users, In Proc. of 18th International Conference on Computer Supported Cooperative Work in Design (CSCWD), IEEE,2014,pp. 609-614.

7. Amendola S., Lodato R., Manzari S., Occhiuzzi C. and Marrocco G., RFID technology for IoT-based personal healthcare in smart spaces, Internet of Things, vol.1, no.2, pp.144-152, 2014

8. Kang Y. and Zhongyi Z., Summarize on Internet of Things and exploration into technical system framework, In Proc. of Symposium on Robotics and Applications (ISRA), June, 2012, pp. 653-656.

9. Ortiz A.M., Hussein D., Park S., Han S.N. and Crespi N., The cluster between internet of things and social networks: Review and research challenges, Internet of Things, vol.1, no.3, pp.206-215, 2014

10. Wan J. Zou, C., Zhou K., Lu R. and Li D., IoT sensing framework with inter-cloud computing capability in vehicular networking, Electronic Commerce Research, vol.14, no.3, pp.389-416, 2014.

11. Domingo M.C., An overview of the Internet of Things for people with disabilities, Journal of Network and Computer Applications, vol.35, no.2, pp.584-596, 2012. 
12. Castellani A., Bui N., Casari P., Rossi M., Shelby Z. and Zorzi M., Architecture and protocols for the internet of things: A case study, In Proc. of 8th IEEE International Conference on Pervasive Computing and Communications Workshops (PERCOM Workshops), Mannheim, March, 2010, pp. 678-683.

13. Bandyopadhyay D. and Sen J., Internet of things: Applications and challenges in technology and standardization, Wireless Personal Communications, vol.58, no.1, pp.49-69, 2011.

14. Sheng Z., Yang S., Yu Y., Vasilakos A., Mccann J. and Leung K., A survey on the ietf protocol suite for the internet of things: Standards, challenges, and opportunities, Wireless Communications, vol.20, no.6, pp.91-98, 2013.

15. Gubbi J., Buyya R., Marusic S. and Palaniswami M., Internet of Things (IoT): A vision, architectural elements, and future directions, Future Generation Computer Systems, vol.29, no.7, pp.1645-1660, 2013.

16. Xu B., Da Xu, L. Cai, H. Xie, C., Hu J. and Bu F., Ubiquitous data accessing method in IoT-based information system for emergency medical services, IEEE Transactions on Industrial Informatics, vol.10, no.2, pp.1578-1586, 2014.

17. Santos A., Macedo J., Costa A. and Nicolau M.J., Internet of things and smart objects for M-health monitoring and control, Procedia Technology, vol.16, 2014, pp.1351-1360.

18. Mitra U., Emken B.A., Lee S., Li M., Rozgic V., Thatte G., Vathsangam H., Zois D.S., Annavaram M., Narayanan S. and Levorato M., KNOWME: a case study in wireless body area sensor network design, IEEE Communications Magazine, vol.50, no.5, pp.116-125, 2012.

19. Information and Communication Technology (ICT) Statistics (2011) ITU. http://www.itu.intITU-Dictindex.html. (Accessed on March 5, 2016)

20. Franklin V, Greene A, Waller A, Greene S, and Pagliari C, 'Patients' Engagement With "Sweet Talk" - A Text Messaging Support System for Young People With Diabetes, Journal Of Medical Internet Research, vol.10, no.2, p. 6, 2008.

21. Kang J.M., Yoo T. and Kim H.C., A wrist-worn integrated health monitoring instrument with a tele-reporting device for telemedicine and tele care, IEEE Transactions on Instrumentation and Measurement, vol. 55, no.5, pp.1655-1661, 2006.

22. Pollonini L., Rajan N.O., Xu S., Madala S. and Dacso C.C., A novel handheld device for use in remote patient monitoring of heart failure patientsDesign and preliminary validation on healthy subjects, Journal of medical systems, vol.36, no.2, pp.653-659, 2012.

23. Kwapisz J.R., Weiss G.M. and Moore S.A., Activity recognition using cell phone accelerometers, ACM Sig KDD Explorations Newsletter, vol.12, no.2, pp.74-82, 2011.

24. Moghaddam R.F., Moghaddam F.F. and Cheriet M., The bluenetwork concept, University of Quebec, Montreal, Canada, pp.1-8, 2011.

25. Miloevi M., Shrove M.T. and Jovanov E., Applications of smartphones for ubiquitous health monitoring and wellbeing management, JITA-Journal of Information Technology and Applications (Banja Luka)-APEIRON, vol.1, no.1, 2011.

26. Oresko J. J., Jin Z., Cheng J., Huang S., Sun Y., Duschl H. and Cheng A.C., A wearable smartphone-based platform for real-time cardiovascular disease detection via electrocardiogram processing, IEEE Transactions on Information Technology in Biomedicine, vol.14, no.3, pp.734-740, 2010.

27. Azumia Instant heart rate. http://www.azumio.com/s/instantheartrate/index.html (Accessed on March 10, 2016)

28. Kumar N., Kaur K., Misra S.C. and Iqbal R., An intelligent RFID-enabled authentication scheme for healthcare applications in vehicular mobile cloud, Peer-to-Peer Networking and Applications, pp.1-17, 2015.

29. Hassanalieragh M., Page A., Soyata T., Sharma, G., Aktas M., Mateos G., Kantarci B. and Andreescu S., Health monitoring and management using internet-of-things (IoT) sensing with cloud based processing: Opportunities and challenges, In Proc. ofIEEE International Conference on Services Computing (SCC), New York, USA, 2015, pp. 285-292.

30. Seales C., Do T., Belyi E. and Kumar S., PHINet: A Plug-n-Play Content-centric Testbed Framework for Health-Internet of Things, In Proc. of IEEE International Conference on Mobile Services (MS), New York, USA, 2015, pp. 368-375. 